\title{
Investigation of Organic Matter at The Micron Scale in Carbonaceous Chondrites: a Spyglass to Study The Early Solar System.
}

\author{
Laurent Remusat ${ }^{1}$, Corentin Le Guillou ${ }^{2}$, Sylvain Bernard ${ }^{1}$, Vassilissa Vinogradoff ${ }^{1}$, Adrian Brearley ${ }^{3}$. \\ 1. IMPMC, UMR CNRS 7590, Sorbonne Universités, UPMC, IRD, MNHN, Paris, France. \\ 2. UMET, UMR CNRS 8207, Univ. Lille1, Villeneuve d'Ascq, France. \\ ${ }^{3}$ Dept. Earth and Planetary Sciences, University of New Mexico, Albuquerque, USA.
}

Carbonaceous chondrites are primitive meteorites formed 4.56 Gy ago. During the first stages of the solar system, they accreted material from the interstellar space that was processed in the disk and mixed with other solar system components. All these materials were likely millimeter to micrometer grains. Their investigation at fine scale thus provides important clues to understand solar system formation. The matrix of CI, CM and CR carbonaceous chondrites contains abundant hydrated minerals, such as clays, and up to $5 \mathrm{wt} . \%$ of organic matter $(\mathrm{OM})$. These meteorites have likely brought volatiles to the early Earth during the late veneer, hence delivering to the Earth potential building blocks of Life. A better understanding of OM contained in chondrites may help to refine scenarios of solar system formation and emergence of Life on Earth. OM and water were likely accreted together as ice grains within asteroids and other planetary embryos, and their abundance is therefore correlated. After accretion, internal heating due to decay of short-lived radionuclides melted the ice and induced fluid circulation that promoted mineralogical and chemical reactions on the asteroidal parent bodies of these chondrites. This aqueous (or hydrothermal) alteration has modified some of the chemical and isotopic signatures that the OM could have acquired during it synthesis, hence blurring the record of protosolar conditions.

A multi technique characterization of $\mathrm{OM}$ at the micrometer and nanometer scales, combining imaging by NanoSIMS (Nano Secondary Ion Mass Spectrometry for elemental and isotopic imaging), STXM (scanning transmission X-ray microscopy, providing spatially resolved XANES spectra, X-ray absorption near edge structure, for molecular characterization) and TEM (transmission electron microscopy, for textural and mineralogical characterization) can be employed to sort OM from other Crich material, and determine its origin and evolution [1,2]. However, OM occurs as micron-sized grains dispersed throughout the matrix and as a diffuse material intimately mixed with hydrated minerals (figure 1); in rare occasions, up to 10 microns large veins may occur. Thanks to the preparation of ultrathin section by FIB (focused ion beam), isotopic and molecular properties of OM can be investigated in the intact petrographic and textural contexts in order to evaluate OM-mineral interactions. This challenging approach provides compelling data to shed new light on the evolution of OM during the hydrothermal alteration on the parent body and disclose its origin.

Using this integrated approach, we have studied the OM in the Orgueil (CI), Murchison (CM), and Renazzo (CR) chondrites, which exhibit various degrees of hydrothermal alteration. In all of them, the deuterium to hydrogen $(\mathrm{D} / \mathrm{H})$ ratio of their $\mathrm{OM}$ shows large heterogeneities, at all scales $(\mathrm{mg}$, nanometer, molecular) $[3,4,5]$. However, there is no clear connection between isotopic composition and local mineralogy. The comparison of the molecular signatures of OM in the three different chondrites clearly shows that increasing hydrothermal alteration increases heterogeneity within the same chondrite [6]. In addition, aromatic and carboxylic carbon contributions tend to increase at the expense of aliphatic carbon. The texture of OM slightly evolves as phyllosilicates grow: the contribution of the diffuse OM increases in more altered chondrites and the size of OM particles tends to decrease. To push further our 
investigations, we studied the Paris chondrite (fig. 1), a unique CM showing two distinct lithologies in the same stone, one being much more altered than the other one. It constitutes a Rosetta stone to disentangle the effects of hydrothermal alteration from starting material heterogeneity. The diffuse OM and organic particles present significant heterogeneity as revealed by XANES: nearby organic particles occurring in the same petrographic context of an altered area exhibit a distinct molecular signature (fig. 1). In addition, the formation of some alteration minerals (e.g. tochilinite) eradicates the OM from some areas (fig. 1), whereas others (e.g. clays) entrap it.

The molecular and isotopic heterogeneities can result from hydrothermal alteration on the asteroidal parent body. A similar organic precursor could have been accreted in all chondrites, and later modified to various degrees related to the intensity of the aqueous alteration [3]. Alternatively, heterogeneities could be inherited from a diversity of organic particles in the early solar system. Before accretion into asteroids, organic grains could have undergone a large array of processes as the result of exposure to different regions of the protosolar disk associated with active turbulence mixing. Furthermore, an interstellar heritage of some presolar organic grains cannot be excluded. The origin of OM in chondrites remains complex to resolve as a consequence of the likely superimposition of these events.

\section{References:}

[1] L. Remusat et al, Geochim. Cosmochim. Acta 96 (2012), p. 319-335.

[2] B.T. De Gregorio et al, Meteorit. Planet. Sci. 46 (2011), p. 1376-1396.

[3] C.M.O'D. Alexander, et al, Geochim. Cosmochim. Acta 74 (2010). p. 4417-4437.

[4] L. Remusat, et al, Earth Planet. Sci. Let. 243 (2006), p. 15-25.

[5] L. Remusat, et al, ApJ 698 (2009), p. 2087-2092.

[6] C. Le Guillou, et al, Geochim. Cosmochim. Acta 131 (2014), p. 368-392.

\section{NanoSIMS}

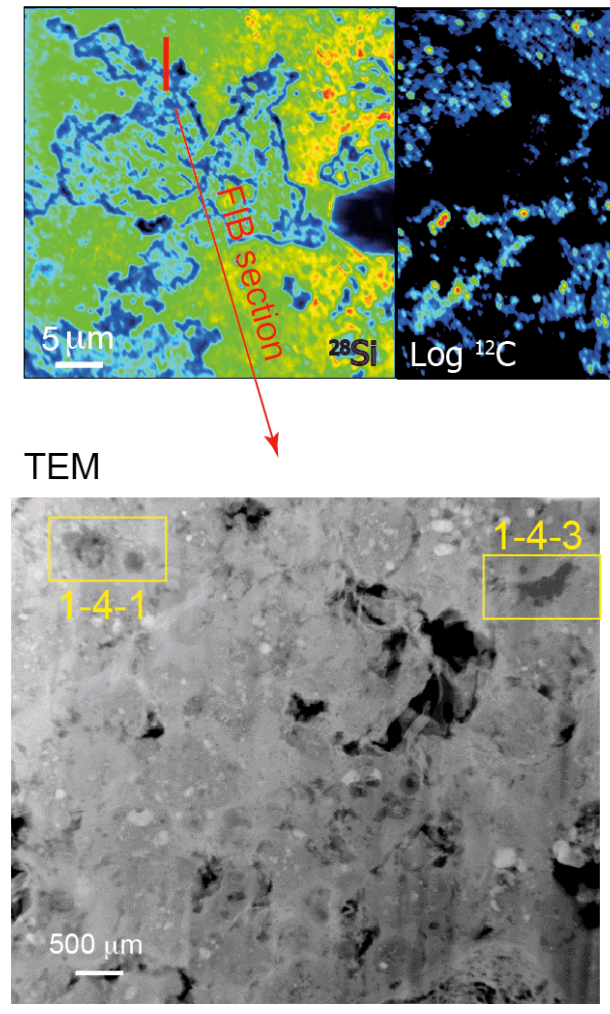

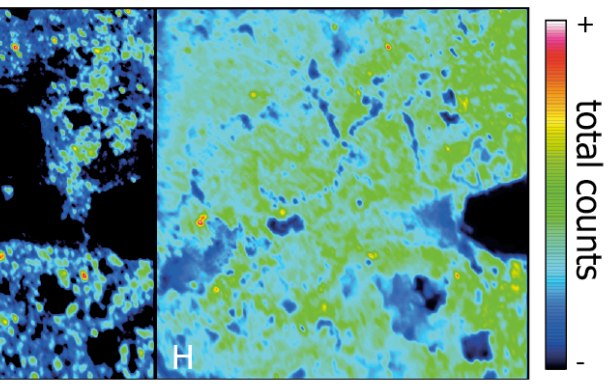

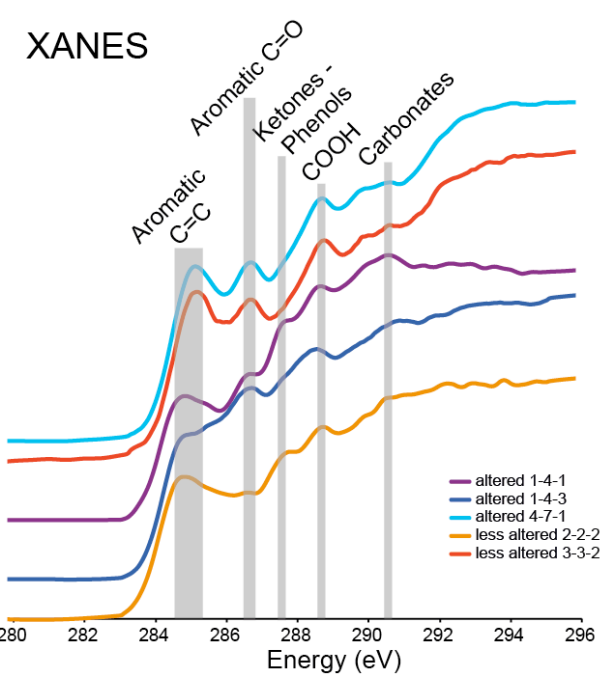

Figure 1. Areas rich in $\mathrm{C}$ and $\mathrm{H}$ in NanoSIMS images are organic particles. Thanks to FIB, a thin section can be extracted and imaged at the nm scale by TEM, to determine the petrology around organic grains (amorphous silicate, phyllosilicate or sulfide). The molecular properties of these grains are investigated by STXM. Here, two grains that occur in similar context show very distinct XANES signature (dark blue and purple spectra). 